\title{
Quantitative Temperature Dependence of Longitudinal Spin Seebeck Effect at High Temperatures
}

\author{
Ken-ichi Uchida, ${ }^{1,2, *}$ Takashi Kikkawa, ${ }^{1}$ Asuka Miura, ${ }^{3}$ Junichiro Shiomi, ${ }^{2,3}$ and Eiji Saitoh ${ }^{1,4,5,6}$ \\ ${ }^{1}$ Institute for Materials Research, Tohoku University, Sendai 980-8577, Japan \\ ${ }^{2}$ PRESTO, Japan Science and Technology Agency, Saitama 332-0012, Japan \\ ${ }^{3}$ Department of Mechanical Engineering, The University of Tokyo, Tokyo 113-8656, Japan \\ ${ }^{4}$ WPI Advanced Institute for Materials Research, Tohoku University, Sendai 980-8577, Japan \\ ${ }^{5}$ CREST, Japan Science and Technology Agency, Tokyo 102-0076, Japan \\ ${ }^{6}$ Advanced Science Research Center, Japan Atomic Energy Agency, Tokai 319-1195, Japan
}

(Received 10 August 2014; published 5 November 2014)

\begin{abstract}
We report temperature-dependent measurements of longitudinal spin Seebeck effects (LSSEs) in $\mathrm{Pt} / \mathrm{Y}_{3} \mathrm{Fe}_{5} \mathrm{O}_{12}$ (YIG)/Pt systems in a high temperature range from room temperature to above the Curie temperature of YIG. The experimental results show that the magnitude of the LSSE voltage in the $\mathrm{Pt} / \mathrm{YIG} / \mathrm{Pt}$ systems rapidly decreases with increasing the temperature and disappears above the Curie temperature. The critical exponent of the LSSE voltage in the Pt/YIG/Pt systems at the Curie temperature is estimated to be 3 , which is much greater than that for the magnetization curve of YIG. This difference highlights the fact that the mechanism of the LSSE cannot be explained in terms of simple static magnetic properties in YIG.
\end{abstract}

DOI: 10.1103/PhysRevX.4.041023

\section{INTRODUCTION}

The Seebeck effect converts a temperature difference into electric voltage in conductors [1]. Since the discovery of the Seebeck effect nearly 200 years ago, it has been studied intensively to realize simple and environmentally friendly energy-conversion technologies [2]. The Seebeck effect has been measured using various materials in a wide temperature range to investigate thermoelectric conversion performance and thermoelectric transport properties. Temperature-dependent measurements in a high temperature range are especially important in the investigation of the Seebeck effect, since thermoelectric devices are often used above room temperature $[3,4]$.

In the field of spintronics [5-7], a spin counterpart of the Seebeck effect-the spin Seebeck effect (SSE)—was recently discovered. The SSE converts a temperature difference into spin voltage in ferromagnetic or ferrimagnetic materials. When a conductor is attached to a magnet under a temperature gradient, the thermally generated spin voltage in the magnet injects a spin current [8-10] into the conductor. Since the SSE occurs not only in metals [11-14] and semiconductors $[15,16]$ but also in insulators $[13,17-32]$, it enables the construction of "insulator-based

\footnotetext{
*kuchida@imr.tohoku.ac.jp
}

Published by the American Physical Society under the terms of the Creative Commons Attribution 3.0 License. Further distribution of this work must maintain attribution to the author(s) and the published article's title, journal citation, and DOI.
Subject Areas: Condensed Matter Physics,

Magnetism, Spintronics thermoelectric generators" [21] in combination with the inverse spin Hall effect (ISHE) [33-37], which was impossible if only the conventional Seebeck effect was used.

The observation of the SSE in insulators has been reported mainly in a longitudinal configuration [13,18-32]. The sample system for measuring the longitudinal SSE (LSSE) is a simple paramagnetic metal (PM)-ferrimagnetic insulator (FI) junction system. In many cases, Pt and $\mathrm{Y}_{3} \mathrm{Fe}_{5} \mathrm{O}_{12}$ (YIG) are used as PM and FI, respectively. When a temperature gradient $\nabla T$ is applied to the PM-FI system perpendicular to the interface, the spin voltage is thermally generated and injects a spin current into the PM along the $\nabla T$ direction owing to thermal spin-pumping mechanism [38-46]. This thermally induced spin current is converted into an electric field $\mathbf{E}_{\mathrm{ISHE}}$ by the ISHE in the PM according to the relation

$$
\mathbf{E}_{\mathrm{ISHE}} \propto \mathbf{J}_{s} \times \boldsymbol{\sigma}
$$

where $\mathbf{J}_{s}$ is the spatial direction of the thermally induced spin current and $\sigma$ is the spin-polarization vector of electrons in the PM, which is parallel to the magnetization $\mathbf{M}$ of FI [see Fig. 1(a)]. By measuring $\mathbf{E}_{\text {ISHE }}$ in the PM, one can detect the LSSE electrically.

In the experimental research on the SSE, temperaturedependent measurements also have been used for investigating various thermospin transport properties, such as phonon-mediated effects $[15,16,39,47]$, correlation between the SSE and magnon excitation $[29,48]$, and effects of metalinsulator phase transition [13]. However, all the experiments 

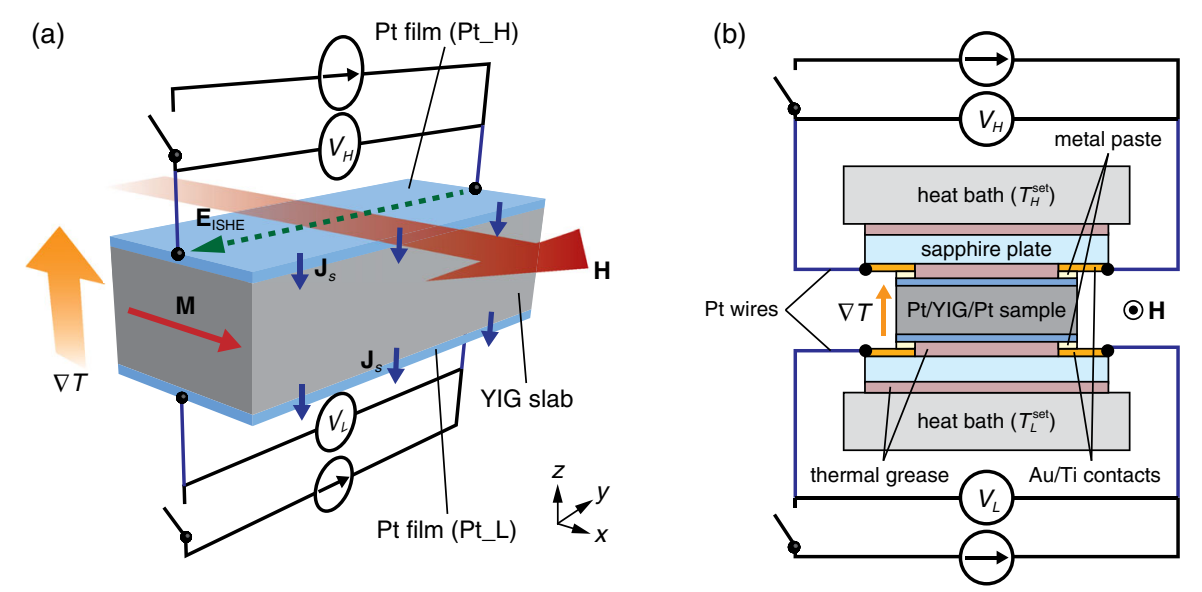

(c)
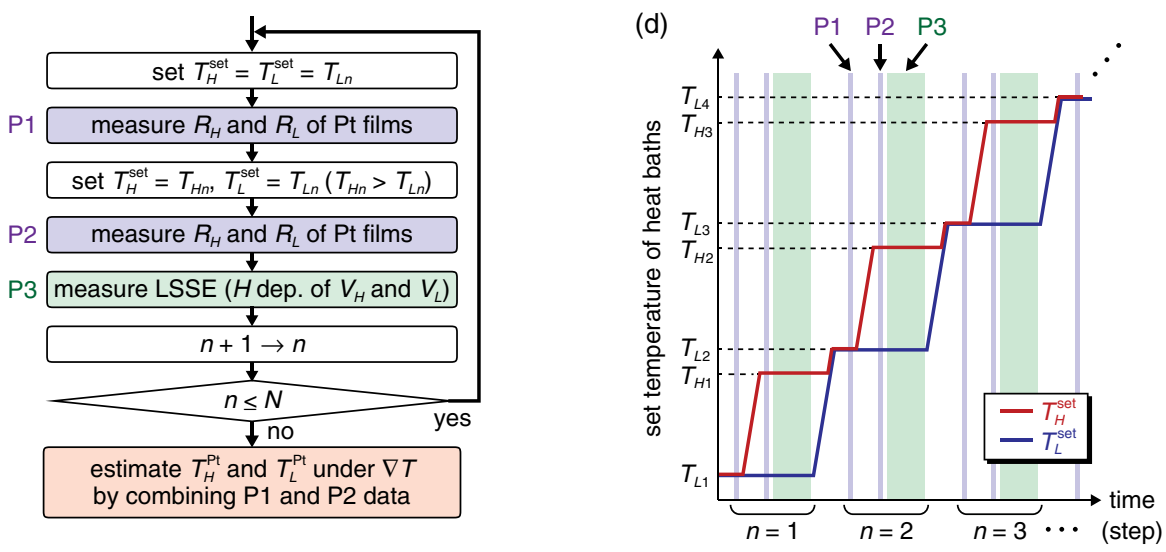

FIG. 1. (a) A schematic illustration of the Pt/YIG/Pt sample. $\nabla T, \mathbf{H}, \mathbf{M}, \mathbf{E}_{\mathrm{ISHE}}$, and $\mathbf{J}_{s}$ denote the temperature gradient, magnetic field (with the magnitude $H$ ), magnetization vector, electric field induced by the ISHE, and spatial direction of the thermally generated spin current, respectively. The electric voltage $V_{H}\left(V_{L}\right)$ and resistance $R_{H}\left(R_{L}\right)$ between the ends of Pt_H (Pt_L) are measured using a nanovolt, micro-ohm meter (Agilent 34420A) in the "dc-voltage" and "2-wire-resistance" modes, respectively. The dc-voltage (2-wire-resistance) mode corresponds to the switch-off (switch-on) state in this schematic illustration. (b) Experimental configuration for measuring the LSSE used in the present study. (c) Flow chart of the measurement processes. (d) Schematic graph of the set temperatures, $T_{H n}$ and $T_{L n}$, of the heat baths as a function of time or the measurement step number $n$. Process P1 is performed under the isothermal condition, while processes P2 and P3 are under the temperature gradient. Before starting processes P1 and P2, we wait for $\sim 30$ min at each $n$ to stabilize the $T_{H}^{\text {set }}$ and $T_{L}^{\text {set }}$ values.

on the SSE to date have been performed around and below room temperature. In this article, we report quantitative temperature-dependent measurements of the LSSE in $\mathrm{Pt} / \mathrm{YIG}$ systems in the high temperature range from room temperature to above the Curie temperature of YIG.

\section{EXPERIMENTAL PROCEDURE}

The sample system used in this study consists of a singlecrystalline YIG slab covered with Pt films. One difference from conventional samples is that the Pt films are put on both the top and bottom surfaces of the YIG slab [Fig. 1(a)], while only the top surface of YIG is covered with a Pt film in conventional samples $[18,23,27,32]$. The lengths of the YIG slab along the $x, y$, and $z$ directions are 3,7 , and $1 \mathrm{~mm}$, respectively. Pt films $10 \mathrm{~nm}$ thick are sputtered on the whole of the $3 \times 7 \mathrm{~mm}^{2}$ (111) surfaces of the YIG. The top and bottom Pt films are electrically insulated from each other because YIG is a very good insulator. Since YIG has a large charge gap of $2.7 \mathrm{eV}$ [49], thermal excitation of charge carriers in YIG is vanishingly small even at the high temperatures.

To attach electrodes to both the Pt films symmetrically and to generate a uniform temperature gradient, we make the configuration shown in Fig. 1(b). Here, the Pt/YIG/Pt sample is sandwiched between two $0.5-\mathrm{mm}$-thick sapphire plates of which the surface is covered with two separated $\mathrm{Au} / \mathrm{Ti}$ contacts. The distance between the two $\mathrm{Au} / \mathrm{Ti}$ contacts is $\sim 6 \mathrm{~mm}$. To extract voltage signals in the $\mathrm{Pt}$ films, both the ends of the Pt films are connected to the $\mathrm{Au} / \mathrm{Ti}$ contacts via sintering metal paste, which can be used up to $900^{\circ} \mathrm{C}$, and thin $\mathrm{Pt}$ wires with diameter of $0.1 \mathrm{~mm}$ are attached to the end of the contacts [see Fig. 1(b)]. The sapphire plates are thermally connected to heat baths of which the temperatures are controlled with an accuracy of $<0.6 \mathrm{~K}$ by using proportional-integral-derivative 
temperature controllers. We attach thermal paste to both the surfaces of the sapphire plates, except for the regions of the $\mathrm{Au} / \mathrm{Ti}$ contacts, to improve the thermal contact [see Fig. 1(b)]. During the measurements of the LSSE, the temperature of the upper heat bath $T_{H}^{\text {set }}$ is set to be higher than that of the lower one $T_{L}^{\text {set }}$. According to the direction of the temperature gradient, hereafter, the top and bottom $\mathrm{Pt}$ films of the Pt/YIG/Pt sample are referred to as "Pt_H" and "Pt_L," respectively. In this setup, we can measure the electric voltage $V_{H}\left(V_{L}\right)$ and resistance $R_{H}\left(R_{L}\right)$ between the ends of Pt_H (Pt_L) without changing electrodes, wiring, and measurement equipment [see also the caption of Fig. 1]. An external magnetic field $\mathbf{H}$ (with the magnitude $H$ ) was applied along the $x$ direction.

To investigate the temperature dependence of the LSSE quantitatively, it is important to estimate the temperature difference between the top and bottom of the YIG sample. However, in conventional experiments, the temperatures of the heat baths, not the sample itself, are usually monitored [20]. Therefore, the measured temperature difference includes the contributions from the interfacial thermal resistance between the sample and heat baths and from small temperature gradients in the sample holders. To avoid this problem, in this study, we use the Pt films not only as spin-current detectors but also as temperature sensors [28,31]; we can know the temperatures of the Pt films from the temperature dependence of the resistance of the films, enabling the estimation of the temperature difference between the top and bottom of the sample during the LSSE measurements.

Figure 1(c) shows the flow chart of the measurement processes. The measurement comprises the three processes $\mathrm{P} 1-\mathrm{P} 3$, and the processes are repeated $N$ times. Hereafter, $T_{H n}$ and $T_{L n}$ denote the set temperatures for each measurement step number $n(=1,2, \ldots, N)$ (see Table I, where the values of $T_{H n}$ and $T_{L n}$ for each $n$ are shown). Process

TABLE I. Set temperatures of the heat baths for each measurement step number $n(\leq N)$. Here, the $T_{H n}$ and $T_{L n}$ values are increased by $10 \mathrm{~K}$ with every $n$ increase, while $T_{H n}-T_{L n}$ is fixed at $8 \mathrm{~K}$. The single dots represent the abbreviation of the $n, T_{H n}$, and $T_{L n}$ values.

\begin{tabular}{lcc}
\hline \hline Step number $n$ & $T_{L n}(\mathrm{~K})$ & $T_{H n}(\mathrm{~K})$ \\
\hline 1 & 290 & 298 \\
2 & 300 & 308 \\
3 & 310 & 318 \\
4 & 320 & 328 \\
5 & 330 & 338 \\
$\cdot$ & $\cdot$ & $\cdot$ \\
$\cdot$ & $\cdot$ & $\cdot$ \\
31 & $\cdot$ & $\cdot$ \\
32 & 590 & 598 \\
33 & 600 & 608 \\
$34(=N)$ & 610 & 618 \\
\hline \hline
\end{tabular}

$\mathrm{P} 1$ is the measurement of the resistance of $\mathrm{Pt} \_\mathrm{H}$ and $\mathrm{Pt} \_\mathrm{L}$, $R_{H}$ and $R_{L}$, in the isothermal condition, where the temperatures of the heat baths are set to the same temperature: $T_{H}^{\mathrm{set}}=T_{L}^{\mathrm{set}}=T_{L n}$. In this isothermal condition, the temperature of the Pt/YIG/Pt sample is uniform and very close to the set temperature of the heat baths irrespective of the presence of the interfacial thermal resistance. Next, we apply a temperature gradient to the $\mathrm{Pt} / \mathrm{YIG} / \mathrm{Pt}$ sample by increasing the temperature of the upper heat bath, where $T_{H}^{\text {set }}=T_{H n}$ and $T_{L}^{\text {set }}=T_{L n}$, with $T_{H n}>T_{L n}$ (see Table I). After waiting until the temperatures are stabilized, we measure the resistance of the Pt films under the temperature gradient: this is process $\mathrm{P} 2$. Processes $\mathrm{P} 1$ and $\mathrm{P} 2$ are performed without applying $\mathbf{H}$. Immediately after process P2, we proceed to process P3; the LSSE, i.e., the $H$ dependence of $V_{H}$ and $V_{L}$, is measured with keeping the magnitude of $T_{H}^{\text {set }}-T_{L}^{\text {set }}$ constant. After finishing the LSSE measurements, we go on to the next step and increase $n$ by 1 , where the values of $T_{H n}$ and $T_{L n}$ are increased as shown in Table I. These measurement processes are summarized in Fig. 1(d).

The calibration method of the sample temperatures is as follows. From process P1, we obtain the temperature $\left(T_{L n}\right)$ dependence of $R_{H}$ and $R_{L}$ under the isothermal condition. By comparing the resistance under the temperature gradient, obtained from process $\mathrm{P} 2$, with the isothermal $R_{H, L}-T_{L n}$ curves, we can calibrate the temperature of the Pt films, $T_{H}^{\mathrm{Pt}}$ and $T_{L}^{\mathrm{Pt}}$, under the temperature gradient, allowing us to estimate the average temperature $T_{\mathrm{av}}$ $\left[=\left(T_{H}^{\mathrm{Pt}}+T_{L}^{\mathrm{Pt}}\right) / 2\right]$ and the temperature difference $\Delta T$ $\left(=T_{H}^{\mathrm{Pt}}-T_{L}^{\mathrm{Pt}}\right)$ in the YIG slab during the LSSE measurements. The $T_{\text {av }}$ and $\Delta T$ values are free from the contributions from the interfacial thermal resistance between the sample and heat baths and from the temperature gradients in the sapphire plates, enabling quantitative evaluation of the LSSE at various temperatures. Although the experimental protocol proposed here cannot estimate the contributions of the interfacial thermal resistance at the Pt/YIG interfaces and temperature gradients in the Pt films, they are negligible compared with the temperature difference applied to the YIG slab [26,32].

\section{RESULTS AND DISCUSSION}

Figures 2(a) and 2(b), respectively, show the $H$ dependence of $V_{H}$ and $V_{L}$ in the Pt/YIG/Pt sample for each step number $n$, measured when $T_{H n}-T_{L n}=8 \mathrm{~K}$. Around room temperature, we observe clear voltage signals in both the Pt films; the signs of $V_{H}$ and $V_{L}$ are reversed in response to the reversal of the magnetization direction of the YIG slab. Since the contribution of anomalous Nernst effects induced by proximity ferromagnetism in $\mathrm{Pt}[50]$ is negligibly small in Pt/YIG systems, the voltage signals observed here are purely due to the LSSE [23,27,32]. The sign of the LSSE voltage in Pt_H is observed to be the same as that in $\mathrm{Pt} \_\mathrm{L}$, a situation consistent with the scenario of 

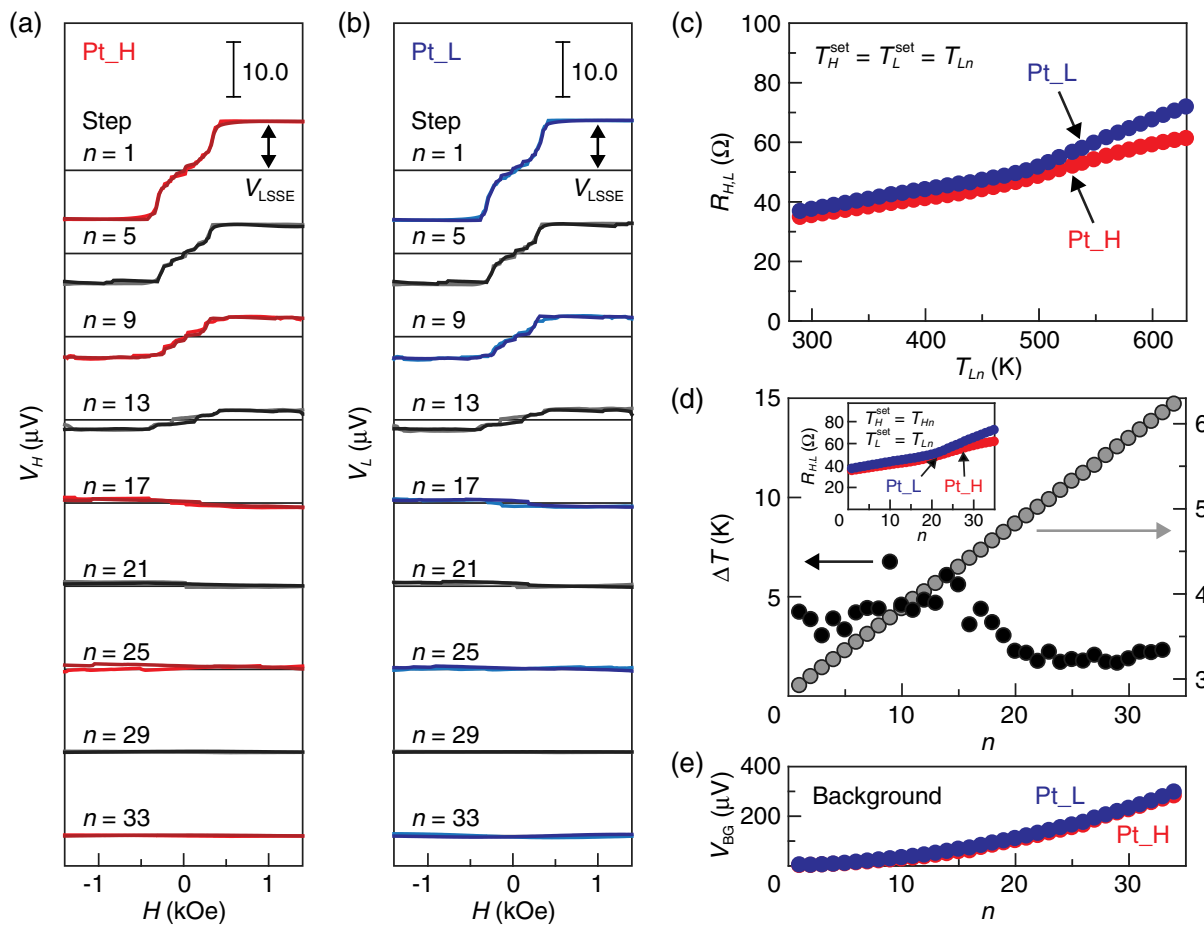

(d)

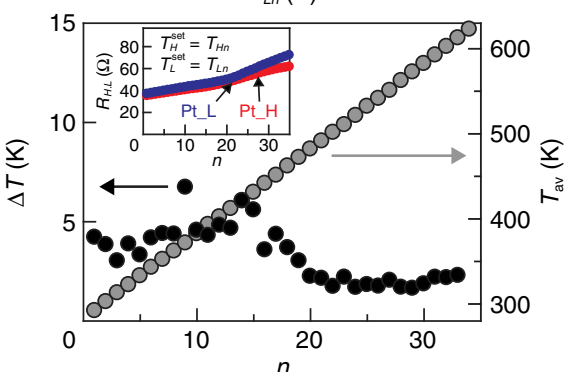

(e)

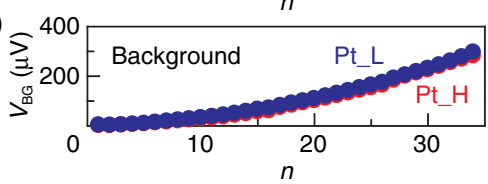

FIG. 2. (a) $H$ dependence of $V_{H}$ for Pt_H in the Pt/YIG/Pt sample for various values of $n$. (b) $H$ dependence of $V_{L}$ for Pt_L. The LSSE voltage $V_{\mathrm{LSSE}}$ for Pt_H (Pt_L) is defined as $V_{H}\left(V_{L}\right)$ at $H=1 \mathrm{kOe}$. (c) $T_{L n}$ dependence of $R_{H}\left(R_{L}\right)$ for Pt_H (Pt_L) in the isothermal condition: $T_{H}^{\mathrm{set}}=T_{L}^{\mathrm{set}}=T_{L n}$. (d) The average temperature $T_{\mathrm{av}}$ and temperature difference $\Delta T$ of the Pt/YIG/Pt sample during the LSSE measurements as a function of $n$. The inset of (d) shows $R_{H}\left(R_{L}\right)$ for Pt_H (Pt_L) as a function of $n$ under the temperature gradient: $T_{H}^{\mathrm{set}}=T_{H n}$ and $T_{L}^{\mathrm{set}}=T_{L n}$ with $T_{H n}>T_{L n}$. (e) The background voltage $V_{\mathrm{BG}}$ for Pt_H and Pt_L as a function of $n$ under the temperature gradient.

the SSE $[38,40,44,51]$. Here, we note that, although the large background voltage $V_{\mathrm{BG}}$ appears due to unavoidable thermopower differences in the wires with increasing $n$ [Fig. 2(e)], the noise level in the $V_{H}$ and $V_{L}$ signals does not increase and the drift of $V_{\mathrm{BG}}$ is small [Figs. 2(a) and 2(b)]. Therefore, we can extract the LSSE voltage simply by measuring the $H$ dependence of $V_{H}$ and $V_{L}$. We also find that the magnitude of the LSSE voltage in the $\mathrm{Pt} / \mathrm{YIG} / \mathrm{Pt}$ sample monotonically decreases with increasing the temperature.

To quantitatively discuss the temperature dependence of the LSSE voltage in the Pt/YIG/Pt sample, we estimate $T_{\text {av }}$ and $\Delta T$ at each step number $n$ by using the method explained in Sec. II. Figure 2(c) shows the $T_{L n}$ dependence of $R_{H}$ and $R_{L}$ for the Pt films measured under the isothermal condition. By combining the isothermal $R_{H, L}-T_{L n}$ curves with the $R_{H}$ and $R_{L}$ data under the temperature gradient [the inset of Fig. 2(d)], we obtain the $T_{\text {av }}$ and $\Delta T$ values at each $n$ [Fig. 2(d)]. Importantly, the calibrated values of $\Delta T$ are dependent on $n$ and always smaller than the temperature difference applied to the heat baths due to the interfacial thermal resistance and temperature gradients in the sapphire plates (note that $T_{H n}-T_{L n}=8 \mathrm{~K}$ for all the measurements, as shown in Table I).
Figure 3(c) shows the LSSE voltage normalized by the calibrated temperature difference applied to the $\mathrm{Pt} / \mathrm{YIG} / \mathrm{Pt}$ sample, $V_{\mathrm{LSSE}} / \Delta T$, as a function of $T_{\mathrm{av}}$. Here, $V_{\mathrm{LSSE}}$ denotes $V_{H}\left(V_{L}\right)$ for Pt_H (Pt_L) at $H=1 \mathrm{kOe}$. We confirm again that the magnitude of $V_{\mathrm{LSSE}} / \Delta T$ monotonically decreases with increasing the temperature and disappears above the Curie temperature $T_{c}$ of YIG, where $T_{c}$ of our YIG slab is experimentally estimated to be $553 \mathrm{~K}$ (see Appendix A). This behavior is observed not only in one sample but also in our different samples as exemplified in Fig. 3(c). Interestingly, the temperature dependence of $V_{\text {LSSE }} / \Delta T$ is significantly different from the magnetization $(4 \pi M)$ curve of the YIG slab [compare Figs. 3(a) and 3(c)]; the magnitude of $V_{\mathrm{LSSE}} / \Delta T$ rapidly decreases with a concave-up shape, while the magnetization curve of YIG exhibits a standard concave-down shape. We also check that the strong temperature dependence of the LSSE voltage in the $\mathrm{Pt} / \mathrm{YIG} / \mathrm{Pt}$ sample cannot be explained by the weak temperature dependence of the thermal conductivity of YIG (see Appendix B). A similar difference in the temperature-dependent data between the ISHE voltage and magnetization is also observed in $\mathrm{Pt} / \mathrm{GaMnAs}$ systems in the measurement of the transverse SSEs $[15,16]$.

The behavior of physical quantities near continuous phase transitions can be described by critical exponents 

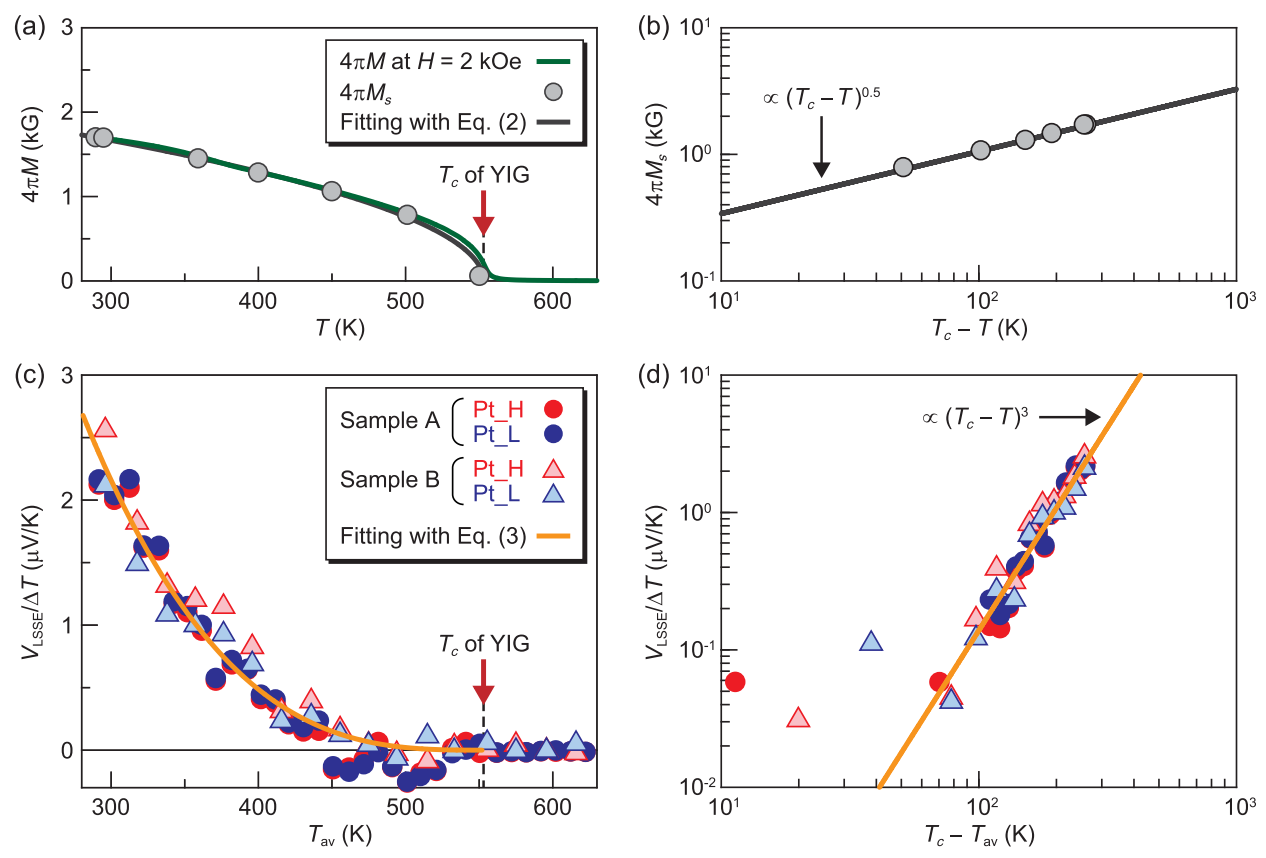

FIG. 3. (a) $T$ dependence of the bulk magnetization $4 \pi M$ of the YIG slab. The green curve shows the $4 \pi M$ data at $H=2 \mathrm{kOe}$, measured with a vibrating sample magnetometer. The gray circles show the saturation magnetization $4 \pi M_{s}$ of the YIG slab. The gray curve is obtained by fitting the $4 \pi M_{s}$ data with Eq. (2). The values of $4 \pi M_{s}$ and Curie temperature $T_{c}(=553 \mathrm{~K})$ of the YIG slab are estimated by using the Arrott plot method (see Appendix A). (b) Double logarithmic plot of the $T_{c}-T$ dependence of $4 \pi M_{s}$ of the YIG slab. (c) $T_{\mathrm{av}}$ dependence of $V_{\mathrm{LSSE}} / \Delta T$ for two different Pt/YIG/Pt samples A (circles) and B (triangles). The experimental results shown in Fig. 2 are measured using the Pt/YIG/Pt sample A. The orange curve is obtained by fitting the $V_{\text {LSSE }} / \Delta T$ data with Eq. (3). (d) Double logarithmic plot of the $T_{c}-T_{\text {av }}$ dependence of $V_{\mathrm{LSSE}} / \Delta T$ for the Pt/YIG/Pt samples $\mathrm{A}$ and B.

in general. Here, we compare the critical exponents for the observed temperature dependences of the LSSE voltage in the $\mathrm{Pt} / \mathrm{YIG} / \mathrm{Pt}$ sample and the magnetization of YIG. First, we check that the magnetization curve of YIG is well reproduced by a standard mean-field model [52]:

$$
4 \pi M_{s}=A\left(T_{c}-T\right)^{0.5}
$$

where the critical exponent is fixed at 0.5 and $A$ is an adjustable parameter [see Figs. 3(a) and 3(b)]. The critical exponent $\gamma$ for the LSSE is estimated by fitting the experimental data in Fig. 3(c) with the following equation:

$$
\frac{V_{\mathrm{LSSE}}}{\Delta T}=S\left(T_{c}-T\right)^{\gamma},
$$

where both $S$ and $\gamma$ are adjustable parameters and $T_{\mathrm{av}}$ is regarded as $T$ for the LSSE data. We find that the observed temperature dependence of $V_{\mathrm{LSSE}} / \Delta T$ for the $\mathrm{Pt} / \mathrm{YIG} / \mathrm{Pt}$ sample is well fitted by Eq. (3) with $\gamma=3$, which is much greater than the critical exponent for the magnetization curve [see also the double logarithmic plot in Fig. 3(d)]. This big difference in the critical exponents between the LSSE and magnetization emphasizes the fact that the LSSE is not attributed solely to static magnetic properties in YIG.

Here, we qualitatively discuss the origin of the temperature dependence of the LSSE voltage in the $\mathrm{Pt} / \mathrm{YIG} / \mathrm{Pt}$ sample. According to the thermal spin-pumping mechanism $[38,44]$ and phenomenological calculation of the ISHE combined with short-circuit effects [53], the magnitude of the LSSE voltage is determined mainly by the following factors: the spin-mixing conductance [54-57] at the Pt/YIG interfaces, spin-diffusion length and spin-Hall angle of Pt, and the difference between an effective magnon temperature in YIG and an effective electron temperature in Pt. Since the LSSE voltage is proportional to the spinmixing conductance [38], it can contribute directly to the observed temperature dependence of the LSSE voltage. Recently, Ohnuma et al. formulated the relation between the spin-mixing conductance and interface $s$ - $d$ interaction at paramagnet-ferromagnet interfaces and predicted that the spin-mixing conductance is proportional to $\left(4 \pi M_{s}\right)^{2}$ of the ferromagnet [58]. By combining this prediction with Eq. (2), the spin-mixing conductance is proportional to $T_{c}-T$, of which the critical exponent $(=1)$ is greater than the exponent for the magnetization curve. Although the temperature dependence of the spin-mixing conductance can explain the facts that the LSSE voltage monotonically decreases with increasing the temperature and disappears at $T_{c}$, it is still much weaker than the observed $\left(T_{c}-T\right)^{3}$ 
dependence of the LSSE voltage. Furthermore, if the spindiffusion length of Pt decreases with increasing the temperature [59], it can also contribute to reducing the LSSE voltage at high temperatures, while the spin-Hall angle of Pt was shown to exhibit weak temperature dependence [60] (note that the magnitude of the ISHE voltage monotonically decreases with decreasing the spin-diffusion length when the spin-Hall angle is constant [53]). The effective magnonelectron temperature difference could also be an important factor, but there is no clear framework to determine its temperature dependence at the present stage. Therefore, more elaborate investigations are necessary for complete understanding of the temperature dependence of the LSSE voltage.

\section{CONCLUSION}

In this study, we report the longitudinal spin Seebeck effects in $\mathrm{Y}_{3} \mathrm{Fe}_{5} \mathrm{O}_{12}$ slabs sandwiched by two Pt films in the high temperature range from room temperature to above the Curie temperature $T_{c}$ of YIG. To investigate the temperature dependence of the LSSE quantitatively, we use the Pt films not only as spin-current detectors but also as temperature sensors. The measurement processes we use enable accurate estimation of the average temperature and temperature difference of the sample, being free from thermal artifacts. We found that the magnitude of the LSSE in the $\mathrm{Pt} / \mathrm{YIG} / \mathrm{Pt}$ sample rapidly decreases with increasing the temperature and disappears above $T_{c}$ of YIG; the observed LSSE voltage exhibits the $\left(T_{c}-T\right)^{3}$ dependence of which the critical exponent $(=3)$ is much greater than that of the magnetization of YIG $(=0.5)$. Although more detailed experimental and theoretical investigations are required to clarify the microscopic origin of this discrepancy, we anticipate that the quantitative temperature-dependent LSSE data at high temperatures will be helpful for obtaining full understanding of the mechanism of the LSSE.

\section{ACKNOWLEDGMENTS}

The authors thank S. Maekawa, H. Adachi, Y. Ohnuma, N. Yokoi, K. Sato, and J. Ohe for valuable discussions and Y. Zhang for his assistance in magnetometry measurements. This work was supported by PRESTO-JST "Phase Interfaces for Highly Efficient Energy Utilization," CREST-JST "Creation of Nanosystems with Novel Functions through Process Integration," Grant-in-Aid for Young Scientists (A) (25707029), Grant-in-Aid for Challenging Exploratory Research (26600067), Grant-inAid for Scientific Research (A) (24244051), Grant-in-Aid for Scientific Research on Innovative Areas "Nano Spin Conversion Science" (26103005) from MEXT, Japan, LCIMR of Tohoku University, the Sumitomo Foundation, the Tanikawa Fund Promotion of Thermal Technology, the
Casio Science Promotion Foundation, and the Iwatani Naoji Foundation.

\section{APPENDIX A: ESTIMATION OF CURIE TEMPERATURE OF YIG}

The Curie temperature $T_{c}$ of the YIG slab used in the present study is estimated from vibrating sample magnetometry and Arrott-plot analysis [52,61]. The inset of Fig. 4(a) shows the $H$ dependence of the magnetization $4 \pi M$ of the YIG slab for various values of $T$. From this result, we obtain the Arrott plots, i.e., $H / 4 \pi M$ dependence of $(4 \pi M)^{2}$, of the YIG slab [see Fig. 4(a)]. The saturation magnetization $4 \pi M_{s}$ of the YIG at each temperature is extracted by extrapolating the $(4 \pi M)^{2}$ data in the highmagnetic-field range to zero field [see red dotted lines in Fig. 4(a)]. As shown in Fig. 4(b), the $T$ dependence of $\left(4 \pi M_{s}\right)^{2}$ of the YIG slab is well fitted by a linear function; the horizontal intercept of the linear fit line corresponds to $T_{c}$. The fitting result shows that the Curie temperature of our YIG slab is $T_{c}=553 \mathrm{~K}$, which is consistent with literature values $[62,63]$.
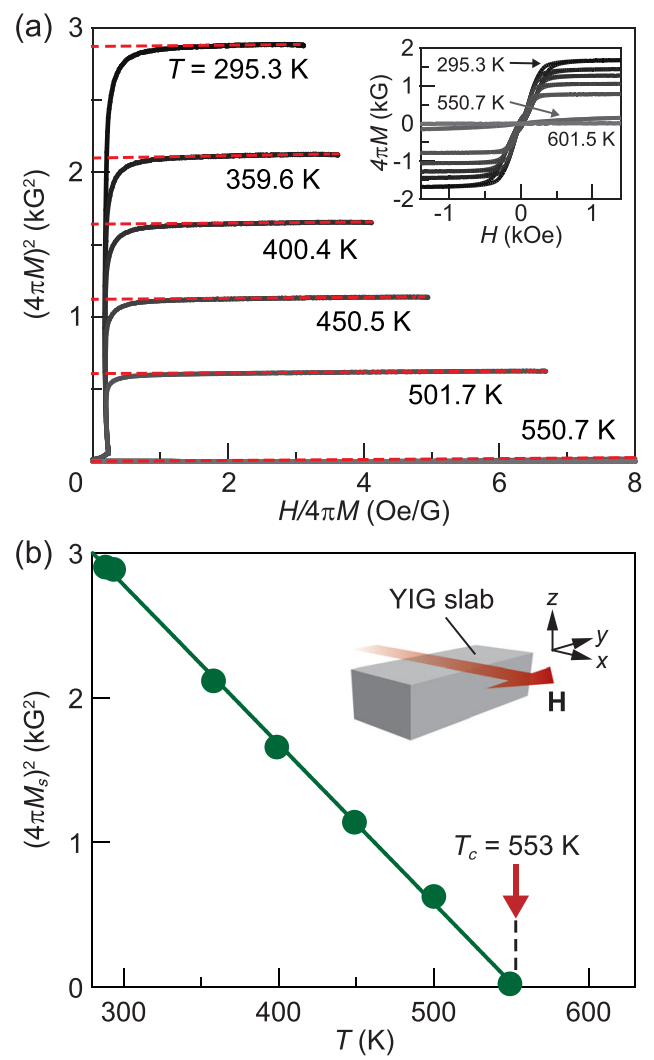

FIG. 4. (a) Arrott plots of the YIG slab for various values of $T$. The inset of (a) shows the $H$ dependence of $4 \pi M$ of the YIG slab for various values of $T$, measured with a vibrating sample magnetometer. The lengths along the $x, y$, and $z$ directions of the YIG slab used for the magnetometry measurements are 3, 7, and $1 \mathrm{~mm}$, respectively. $\mathbf{H}$ is applied along the $x$ direction. (b) $T$ dependence of $\left(4 \pi M_{s}\right)^{2}$ of the YIG slab. 


\section{APPENDIX B: TEMPERATURE DEPENDENCE OF THERMAL CONDUCTIVITY OF YIG}

Figure 5(a) shows the thermal conductivity $\kappa$ of the YIG slab used in the present study as a function of $T$. The $\kappa$ values are obtained by the combination of thermal diffusivity measured by a laser-flash method and specific heat $C$ measured by a differential scanning calorimetry. Here, we measure the thermal diffusivity along the [111] direction of the single-crystalline YIG slab, which is parallel to the $\nabla T$ direction in the LSSE setup. As shown in Fig. 5(b), the measured $C$ values are consistent with the Dulong-Petit (DP) law [1]; the difference of the $C$ values from the DP specific heat of YIG, $C_{\mathrm{DP}}=0.676 \mathrm{~J} / \mathrm{gK}$, is less than $10 \%$ of $C_{\mathrm{DP}}$ for $T>350 \mathrm{~K}$. The observed $T$ dependence of $\kappa$ is well fitted by $\kappa \propto T^{-1}$, indicating that the thermal conductivity of the YIG is dominated by phonons in this temperature range [see also the inset of Fig. 5(a)]. The $\kappa$ value at $300 \mathrm{~K}$ is consistent with literature values [64-66]. We also confirm that the $T$ dependence of $\kappa$ is much weaker than that of the LSSE voltage in the $\mathrm{Pt} / \mathrm{YIG} / \mathrm{Pt}$ sample [compare Figs. 3(c) and 5(a)] and shows no anomaly around $T_{c}$.
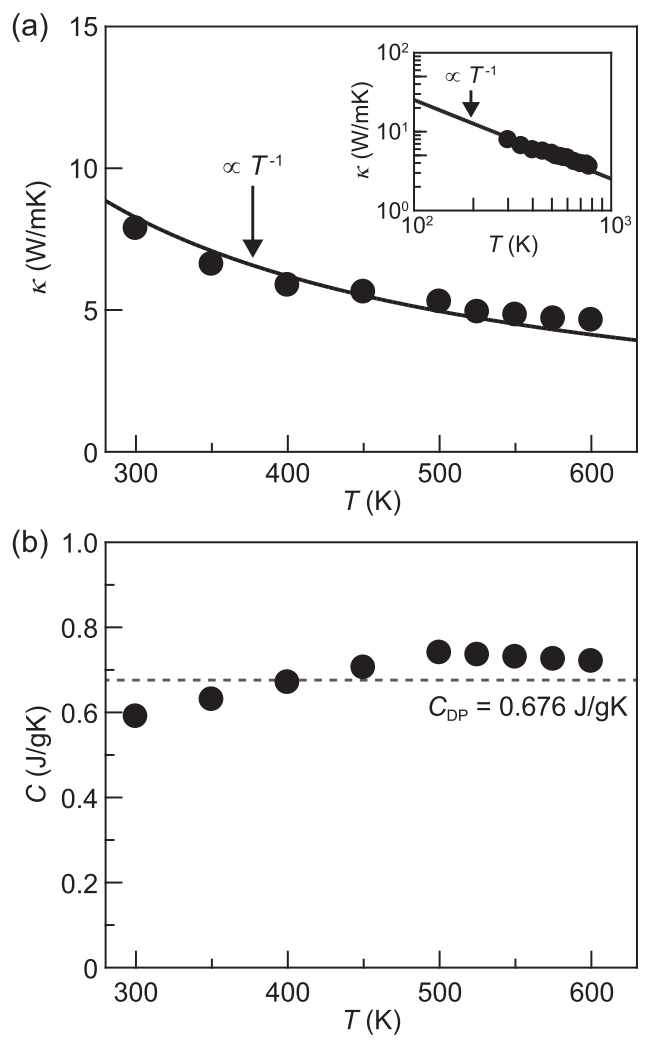

FIG. 5. (a) $T$ dependence of the thermal conductivity $\kappa$ of the YIG slab. The black circles show the measured thermal conductivity and the black curve shows the fitting of the experimental data with $\kappa=K T^{-1}$, where $K$ is an adjustable parameter. The inset shows the double logarithmic plot of the $T$ dependence of $\kappa$. (b) $T$ dependence of the specific heat $C$ of the YIG slab. The gray dotted line shows the DP specific heat of YIG.
[1] N. W. Ashcroft and N.D. Mermin, Solid State Physics (Saunders College, Philadelphia, 1976).

[2] S. B. Riffat and X. Ma, Thermoelectrics: A Review of Present and Potential Applications, Appl. Therm. Eng. 23, 913 (2003).

[3] J. P. Heremans, V. Jovovic, E. S. Toberer, A. Saramat, K. Kurosaki, A. Charoenphakdee, S. Yamanaka, and G. J. Snyder, Enhancement of Thermoelectric Efficiency in PbTe by Distortion of the Electronic Density of States, Science 321, 554 (2008).

[4] L.-D. Zhao, S.-H. Lo, Y. Zhang, H. Sun, G. Tan, C. Uher, C. Wolverton, V. P. Dravid, and M. G. Kanatzidis, Ultralow Thermal Conductivity and High Thermoelectric Figure of Merit in SnSe Crystals, Nature (London) 508, 373 (2014).

[5] S. A. Wolf, D. D. Awschalom, R. A. Buhrman, J. M. Daughton, S. von Molnar, M. L. Roukes, A. Y. Chtchelkanova, and D. M. Treger, Spintronics: A Spin-Based Electronics Vision for the Future, Science 294, 1488 (2001).

[6] I. Žutić, J. Fabian, and S. Das Sarma, Spintronics: Fundamentals and Applications, Rev. Mod. Phys. 76, 323 (2004).

[7] Concepts in Spin Electronics, edited by S. Maekawa (Oxford University Press, Oxford, England, 2006).

[8] J. C. Slonczewski, Current-Driven Excitation of Magnetic Multilayers, J. Magn. Magn. Mater. 159, L1 (1996).

[9] Spin Current, edited by S. Maekawa, E. Saitoh, S. O. Valenzuela, and T. Kimura (Oxford University Press, Oxford, England, 2012).

[10] S. Maekawa, H. Adachi, K. Uchida, J. Ieda, and E. Saitoh, Spin Current: Experimental and Theoretical Aspects, J. Phys. Soc. Jpn. 82, 102002 (2013).

[11] K. Uchida, S. Takahashi, K. Harii, J. Ieda, W. Koshibae, K. Ando, S. Maekawa, and E. Saitoh, Observation of the Spin Seebeck Effect, Nature (London) 455, 778 (2008).

[12] S. Bosu, Y. Sakuraba, K. Uchida, K. Saito, T. Ota, E. Saitoh, and K. Takanashi, Spin Seebeck Effect in Thin Films of the Heusler Compound $\mathrm{Co}_{2} \mathrm{MnSi}$, Phys. Rev. B 83, 224401 (2011).

[13] R. Ramos, T. Kikkawa, K. Uchida, H. Adachi, I. Lucas, M. H. Aguirre, P. Algarabel, L. Morellón, S. Maekawa, E. Saitoh, and M. R. Ibarra, Observation of the Spin Seebeck Effect in Epitaxial $\mathrm{Fe}_{3} \mathrm{O}_{4}$ Thin Films, Appl. Phys. Lett. 102, 072413 (2013).

[14] S. H. Wang, L. K. Zou, J. W. Cai, B. G. Shen, and J. R. Sun, Transverse Thermoelectric Effects in Platinum Strips on Permalloy Films, Phys. Rev. B 88, 214304 (2013).

[15] C. M. Jaworski, J. Yang, S. Mack, D. D. Awschalom, J. P. Heremans, and R.C. Myers, Observation of the SpinSeebeck Effect in a Ferromagnetic Semiconductor, Nat. Mater. 9, 898 (2010).

[16] C. M. Jaworski, J. Yang, S. Mack, D. D. Awschalom, R. C. Myers, and J. P. Heremans, Spin-Seebeck Effect: A Phonon Driven Spin Distribution, Phys. Rev. Lett. 106, 186601 (2011).

[17] K. Uchida, J. Xiao, H. Adachi, J. Ohe, S. Takahashi, J. Ieda, T. Ota, Y. Kajiwara, H. Umezawa, H. Kawai, G. E. W. Bauer, S. Maekawa, and E. Saitoh, Spin Seebeck Insulator, Nat. Mater. 9, 894 (2010).

[18] K. Uchida, H. Adachi, T. Ota, H. Nakayama, S. Maekawa, and E. Saitoh, Observation of Longitudinal Spin-Seebeck Effect in Magnetic Insulators, Appl. Phys. Lett. 97, 172505 (2010). 
[19] M. Weiler, M. Althammer, F. D. Czeschka, H. Huebl, M. S. Wagner, M. Opel, I.-M. Imort, G. Reiss, A. Thomas, R. Gross, and S. T. B. Goennenwein, Local Charge and Spin Currents in Magnetothermal Landscapes, Phys. Rev. Lett. 108, 106602 (2012).

[20] K. Uchida, T. Ota, H. Adachi, J. Xiao, T. Nonaka, Y. Kajiwara, G. E. W. Bauer, S. Maekawa, and E. Saitoh, Thermal Spin Pumping and Magnon-Phonon-Mediated Spin-Seebeck Effect, J. Appl. Phys. 111, 103903 (2012).

[21] A. Kirihara, K. Uchida, Y. Kajiwara, M. Ishida, Y. Nakamura, T. Manako, E. Saitoh, and S. Yorozu, SpinCurrent-Driven Thermoelectric Coating, Nat. Mater. 11, 686 (2012).

[22] D. Qu, S. Y. Huang, J. Hu, R. Wu, and C. L. Chien, Intrinsic Spin Seebeck Effect in Au/YIG, Phys. Rev. Lett. 110, 067206 (2013).

[23] T. Kikkawa, K. Uchida, Y. Shiomi, Z. Qiu, D. Hou, D. Tian, H. Nakayama, X.-F. Jin, and E. Saitoh, Longitudinal Spin Seebeck Effect Free from the Proximity Nernst Effect, Phys. Rev. Lett. 110, 067207 (2013).

[24] D. Meier, T. Kuschel, L. Shen, A. Gupta, T. Kikkawa, K. Uchida, E. Saitoh, J.-M. Schmalhorst, and G. Reiss, Thermally Driven Spin and Charge Currents in Thin $\mathrm{NiFe}_{2} \mathrm{O}_{4}$ /Pt Films, Phys. Rev. B 87, 054421 (2013).

[25] K. Uchida, T. Nonaka, T. Kikkawa, Y. Kajiwara, and E. Saitoh, Longitudinal Spin Seebeck Effect in Various Garnet Ferrites, Phys. Rev. B 87, 104412 (2013).

[26] M. Schreier, A. Kamra, M. Weiler, J. Xiao, G. E. W. Bauer, R. Gross, and S. T. B. Goennenwein, Magnon, Phonon, and Electron Temperature Profiles and the Spin Seebeck Effect in Magnetic Insulator/Normal Metal Hybrid Structures, Phys. Rev. B 88, 094410 (2013).

[27] T. Kikkawa, K. Uchida, S. Daimon, Y. Shiomi, H. Adachi, Z. Qiu, D. Hou, X.-F. Jin, S. Maekawa, and E. Saitoh, Separation of Longitudinal Spin Seebeck Effect from Anomalous Nernst Effect: Determination of the Origin of Transverse Thermoelectric Voltage in Metal/Insulator Junctions, Phys. Rev. B 88, 214403 (2013).

[28] M. Schreier, N. Roschewsky, E. Dobler, S. Meyer, H. Huebl, R. Gross, and S.T.B. Goennenwein, Current Heating Induced Spin Seebeck Effect, Appl. Phys. Lett. 103, 242404 (2013).

[29] S. M. Rezende, R. L. Rodríguez-Suárez, R. O. Cunha, A. R. Rodrigues, F. L. A. Machado, G. A. Fonseca Guerra, J. C. Lopez Ortiz, and A. Azevedo, Magnon Spin-Current Theory for the Longitudinal Spin-Seebeck Effect, Phys. Rev. B 89, 014416 (2014).

[30] N. Roschewsky, M. Schreier, A. Kamra, F. Schade, K. Ganzhorn, S. Meyer, H. Huebl, S. Geprägs, R. Gross, and S. T. B. Goennenwein, Time Resolved Spin Seebeck Effect Experiments, Appl. Phys. Lett. 104, 202410 (2014).

[31] M. Agrawal, V. I. Vasyuchka, A. A. Serga, A. Kirihara, P. Pirro, T. Langner, M. B. Jungfleisch, A. V. Chumak, E. T. Papaioannou, and B. Hillebrands, Role of Bulk-Magnon Transport in the Temporal Evolution of the Longitudinal Spin-Seebeck Effect, Phys. Rev. B 89, 224414 (2014).

[32] K. Uchida, M. Ishida, T. Kikkawa, A. Kirihara, T. Murakami, and E. Saitoh, Longitudinal Spin Seebeck Effect: from Fundamentals to Applications, J. Phys. Condens. Matter 26, 343202 (2014).

[33] A. Azevedo, L. H. Vilela Leão, R. L. Rodriguez-Suarez, A. B. Oliveira, and S. M. Rezende, dc Effect in Ferromagnetic Resonance: Evidence of the Spin-Pumping Effect?, J. Appl. Phys. 97, 10C715 (2005).

[34] E. Saitoh, M. Ueda, H. Miyajima, and G. Tatara, Conversion of Spin Current into Charge Current at Room Temperature: Inverse Spin-Hall Effect, Appl. Phys. Lett. 88, 182509 (2006).

[35] S. O. Valenzuela and M. Tinkham, Direct Electronic Measurement of the Spin Hall Effect, Nature (London) 442, 176 (2006).

[36] M. V. Costache, M. Sladkov, S. M. Watts, C. H. van der Wal, and B. J. van Wees, Electrical Detection of Spin Pumping due to the Precessing Magnetization of a Single Ferromagnet, Phys. Rev. Lett. 97, 216603 (2006).

[37] T. Kimura, Y. Otani, T. Sato, S. Takahashi, and S. Maekawa, Room-Temperature Reversible Spin Hall Effect, Phys. Rev. Lett. 98, 156601 (2007).

[38] J. Xiao, G. E. W. Bauer, K. Uchida, E. Saitoh, and S. Maekawa, Theory of Magnon-Driven Spin Seebeck Effect, Phys. Rev. B 81, 214418 (2010).

[39] H. Adachi, K. Uchida, E. Saitoh, J. Ohe, S. Takahashi, and S. Maekawa, Gigantic Enhancement of Spin Seebeck Effect by Phonon Drag, Appl. Phys. Lett. 97, 252506 (2010).

[40] H. Adachi, J. I. Ohe, S. Takahashi, and S. Maekawa, LinearResponse Theory of Spin Seebeck Effect in Ferromagnetic Insulators, Phys. Rev. B 83, 094410 (2011).

[41] J. I. Ohe, H. Adachi, S. Takahashi, and S. Maekawa, Numerical Study on the Spin Seebeck Effect, Phys. Rev. B 83, 115118 (2011).

[42] S. S.-L. Zhang and S. Zhang, Spin Convertance at Magnetic Interfaces, Phys. Rev. B 86, 214424 (2012).

[43] Y. Ohnuma, H. Adachi, E. Saitoh, and S. Maekawa, Spin Seebeck Effect in Antiferromagnets and Compensated Ferrimagnets, Phys. Rev. B 87, 014423 (2013).

[44] H. Adachi, K. Uchida, E. Saitoh, and S. Maekawa, Theory of the Spin Seebeck Effect, Rep. Prog. Phys. 76, 036501 (2013).

[45] H. Adachi and S. Maekawa, Linear-Response Theory of the Longitudinal Spin Seebeck Effect, J. Korean Phys. Soc. 62, 1753 (2013).

[46] S. Hoffman, K. Sato, and Y. Tserkovnyak, Landau-Lifshitz. Theory of the Longitudinal Spin Seebeck Effect, Phys. Rev. B 88, 064408 (2013).

[47] K. Uchida, H. Adachi, T. An, T. Ota, M. Toda, B. Hillebrands, S. Maekawa, and E. Saitoh, Long-Range Spin Seebeck Effect and Acoustic Spin Pumping, Nat. Mater. 10, 737 (2011).

[48] S. R. Boona and J. P. Heremans, Magnon Thermal Mean Free Path in Yttrium Iron Garnet, Phys. Rev. B 90, 064421 (2014).

[49] Y. Kajiwara, K. Harii, S. Takahashi, J. Ohe, K. Uchida, M. Mizuguchi, H. Umezawa, H. Kawai, K. Ando, K. Takanashi, S. Maekawa, and E. Saitoh, Transmission of Electrical Signals by Spin-Wave Interconversion in a Magnetic Insulator, Nature (London) 464, 262 (2010).

[50] S. Y. Huang, X. Fan, D. Qu, Y. P. Chen, W. G. Wang, J. Wu, T. Y. Chen, J. Q. Xiao, and C. L. Chien, Transport Magnetic 
Proximity Effects in Platinum, Phys. Rev. Lett. 109, 107204 (2012).

[51] Note that the sign of the SSE-induced spin voltage is reversed between the higher- and lower- temperature ends of the ferromagnet $[38,40,44]$. Therefore, in the Pt/YIG/Pt samples used in the present study, the spin voltage in YIG injects a spin current into $\mathrm{Pt}$ on one side, while it ejects a spin current from $\mathrm{Pt}$ on the other side; the direction of $\mathbf{J}_{s}$ in Pt_H is the same as that in Pt_L in the longitudinal configuration.

[52] S. Chikazumi, Physics of Ferromagnetism, 2nd ed. (Oxford University, Oxford, England, 1997).

[53] H. Nakayama, K. Ando, K. Harii, T. Yoshino, R. Takahashi, Y. Kajiwara, K. Uchida, Y. Fujikawa, and E. Saitoh, Geometry Dependence on Inverse Spin Hall Effect Induced by Spin Pumping in $\mathrm{Ni}_{81} \mathrm{Fe}_{19} / \mathrm{Pt}$ Films, Phys. Rev. B 85, 144408 (2012).

[54] Y. Tserkovnyak, A. Brataas, G. E. W. Bauer, and B. I. Halperin, Nonlocal Magnetization Dynamics in Ferromagnetic Heterostructures, Rev. Mod. Phys. 77, 1375 (2005).

[55] X. Jia, K. Liu, K. Xia, and G. E. W. Bauer, Spin Transfer Torque on Magnetic Insulators, Europhys. Lett. 96, 17005 (2011).

[56] M. Weiler, M. Althammer, M. Schreier, J. Lotze, M. Pernpeintner, S. Meyer, H. Huebl, R. Gross, A. Kamra, J. Xiao, Y.-T. Chen, H. Jiao, G. E. W. Bauer, and S. T. B. Goennenwein, Experimental Test of the Spin Mixing Interface Conductivity Concept, Phys. Rev. Lett. 111, 176601 (2013).
[57] Z. Qiu, K. Ando, K. Uchida, Y. Kajiwara, R. Takahashi, H. Nakayama, T. An, Y. Fujikawa, and E. Saitoh, Spin Mixing Conductance at a Well-Controlled Platinum/Yttrium Iron Garnet Interface, Appl. Phys. Lett. 103, 092404 (2013).

[58] Y. Ohnuma, H. Adachi, E. Saitoh, and S. Maekawa, Enhanced dc Spin Pumping into a Fluctuating Ferromagnet near $T_{c}$, Phys. Rev. B 89, 174417 (2014).

[59] S. R. Marmion, M. Ali, M. McLaren, D. A. Williams, and B. J. Hickey, Temperature Dependence of Spin Hall Magnetoresistance in Thin YIG/Pt Films, Phys. Rev. B 89, 220404(R) (2014).

[60] L. Vila, T. Kimura, and Y. C. Otani, Evolution of the Spin Hall Effect in Pt Nanowires: Size and Temperature Effects, Phys. Rev. Lett. 99, 226604 (2007).

[61] A. Arrott, Criterion for Ferromagnetism from Observations of Magnetic Isotherms, Phys. Rev. 108, 1394 (1957).

[62] M. A. Gilleo and S. Geller, Magnetic and Crystallographic Properties of Substituted Yttrium-Iron Garnet, $3 \mathrm{Y}_{2} \mathrm{O}_{3} \cdot x \mathrm{M}_{2} \mathrm{O}_{3} \cdot(5-x) \mathrm{Fe}_{2} \mathrm{O}_{3}$, Phys. Rev. 110, 73 (1958).

[63] E. E. Anderson, Molecular Field Model and the Magnetization of YIG, Phys. Rev. 134, A1581 (1964).

[64] G. A. Slack and D. W. Oliver, Thermal Conductivity of Garnets and Phonon Scattering by Rare-Earth Ions, Phys. Rev. B 4, 592 (1971).

[65] N. P. Padture and P. G. Klemens, Low Thermal Conductivity in Garnets, J. Am. Ceram. Soc. 80, 1018 (1997).

[66] A. M. Hofmeister, Thermal Diffusivity of Garnets at High Temperature, Phys. Chem. Miner. 33, 45 (2006). 